\title{
Caracterizações microbiológica e físico-química de pólens armazenados por abelhas sem ferrão
}

\author{
Microbiological and physicochemical characterization of the pollen \\ stored by stingless bees
}

\author{
Marivalda Figueredo Santa Bárbara1* (1), Cerilene Santiago Machado', Geni da Silva Sodré1, \\ Fabiane de Lima Silva', Carlos Alfredo Lopes de Carvalho' \\ ${ }^{1}$ Universidade Federal do Recôncavo da Bahia (UFRB), Centro de Ciências Agrárias, Ambientais e Biológicas, Cruz das Almas/BA - Brasil
}

\section{${ }^{*}$ Corresponding Author}

Marivalda Figueredo Santa Bárbara, Universidade Federal do Recôncavo da Bahia (UFRB), Centro de Ciências Agrárias, Ambientais e Biológicas, Rua Rui Barbosa, 710, Centro, CEP: 44380-000, Cruz das Almas/BA - Brasil, e-mail: marivaldafsb@gmail.com

Cite as: Microbiological and physicochemical characterization of the pollen stored by stingless bees. Braz. J. Food Technol., v. 21, e2017180, 2018.

Received: Nov. 21, 2017; Accepted: June 26, 2018

\section{Resumo}

O estudo foi realizado com o objetivo de verificar tanto a condição microbiológica quanto as diferenças físico-químicas existentes em pólens armazenados por diferentes espécies de abelhas sem ferrão no Nordeste do Brasil. Os pólens armazenados foram coletados nas colônias mantidas em meliponários das seguintes espécies: Melipona subnitida, M.scutellaris, M. mandacaia, Scaptotrigona sp. e Frieseomellita varia. Com relação às análises de qualidade microbiológica, as mesmas foram realizadas em micro-organismos indicadores de qualidade comercial (mesófilos, bolores e leveduras), qualidade sanitária (Staphylococcus coagulase positiva, coliformes fecais e Escherichia coll), e de segurança (esporos de Clostridium sulfito redutores e Salmonella spp.). Para os parâmetros físico-químicos, os mesmos foram avaliados de acordo com a exigência do Regulamento Técnico (RT) de qualidade para pólen apícola, e também para compostos fenólicos. Todas as amostras apresentaram condição microbiológica de acordo com as recomendações aceitáveis para o consumo humano. Os resultados confirmaram as diferenças na composição físico-química do pólen armazenado das cinco espécies; no entanto, houve formação de três grupos de pólens armazenados com base nos parâmetros físico-químicos. As amostras da espécie M. scutellaris apresentaram conteúdo de compostos fenólicos cinco vezes mais elevado aos outros méis das espécies avaliadas. Logo, este estudo permitiu conhecer a composição dos pólens armazenados por abelhas sem ferrão, por conseguinte reforçando a necessidade de reformular o Regulamento Técnico (RT) existente no Brasil para pólen apícola, de forma a abranger os pólens armazenados das espécies nativas.

Palavras-chave: Compostos fenólicos; Flavonoides; Meliponini; Semiárido.

\section{Abstract}

The present study aimed at checking the microbiological condition and physicochemical differences of the pollen stored by different stingless bee species in the north east of Brazil. The stored pollen was collected from apiaries of colonies of the species Melipona subnitida, M.scutellaris, M. mandacaia, Scaptotrigona sp. and Frieseomellita varia. The microbiological quality was analysed for the main microbial indicators of commercial quality (mesophiles, moulds, and yeasts), sanitary quality (coagulase positive Staphylococcus, faecal coliforms and Escherichia coli) and safety (sulphite-reducing Clostridium spores and Salmonella). The physicochemical parameters evaluated were those required by the Brazilian Technical Regulation for Bee Pollen Quality, as well as the phenolic compounds. The microbiological condition of all the samples was within the recommended levels for human consumption. The results confirmed differences in the physicochemical compositions of the pollens stored by the five species studied, forming three groups according to the physicochemical parameters. The samples of the species $M$. Scutellaris showed five times more phenolic compounds than the pollens of the other species assessed. The present study allowed for knowledge of the composition of the pollens stored by stingless bees, reinforcing the need for reformulation of the current Technical Regulation for Bee Pollen in order to include the pollen stored by native species.

Keywords: Phenolic compounds; Flavonoids; Meliponini; Semiarid. 


\section{Introdução}

As espécies de abelhas sem ferrão da Tribo Meliponini possuem larga criação na região Nordeste do Brasil e apresentam importância econômica. Entre as espécies, destacam-se Melipona (Melipona) quadrifasciata Lepeletier (1936), Melipona (Michmelia) scutellaris Latreille (1811), Melipona (Michmelia) seminigra Friese (1903), Melipona (Michmelia) mondury Smith (1863), Tetragonisca angustula Latreille (1811), Trigona spinipes Fabricius (1793), Scaptotrigona depilis Moure (1942), entre outras (NOGUEIRA-NETO, 1997; CAMARGO; PEDRO, 2013). Estas abelhas possuem características distintas das espécies do gênero Apis, especialmente por terem o ferrão atrofiado (VIT, 2013).

De fato, os pólens armazenados pelas abelhas compreendem uma coleção de grãos de pólen coletados de diversas fontes botânicas, que se mistura ao néctar e também às secreções das glândulas hipofaríngeas, que contêm enzimas $\beta$-glicosidases (CARPES et al., 2009). Estudos comprovaram que o pólen recolhido pelas abelhas tem melhor composição nutricional e maior atividade biológica, quando comparado ao mesmo pólen recolhido diretamente da planta (CHANTARUDEE et al., 2012). Com base nessas características, o pólen pode ser considerado um suplemento terapêutico, nutricional e promissor alimento natural; inclusive, suas propriedades biológicas funcionais são conferidas pelo alto teor de flavonoides e polifenóis e também possuem a capacidade de eliminar radicais livres (DENISOW; DENISOW-PIETRZYK, 2016).
Portanto, os constituintes químicos dos pólens podem ser benéficos na prevenção de doenças em que os radicais livres estão envolvidos, e também pelo fato de serem potentes agentes antibacterianos (ISIDOROV et al., 2015), antioxidantes naturais (DE FLORIO ALMEIDA et al., 2017) e de atuarem como quimiopreventivos (OMAR et al., 2016). Devido a sua importância, estudos direcionados para as caracterizações química e biológica têm sido realizados com os pólens armazenados de diferentes espécies de abelhas, incluindo as abelhas sem ferrão (VIT et al., 2016; REBELO et al., 2016).

Referente à grande diversidade de espécies de abelhas sem ferrão existentes no Brasil, são necessários estudos para a caracterização dos pólens armazenados nessas espécies, visando contribuir na reformulação do Regulamento Técnico (RT) para Pólen Apícola (PA).

Neste contexto, o objetivo desse estudo foi analisar a condição microbiológica e as características físico-químicas dos pólens armazenados por diferentes espécies de abelha sem ferrão com criação no Nordeste do Brasil.

\section{Material e métodos}

Foram avaliadas 19 amostras de pólens armazenados de cinco espécies de abelha sem ferrão dos seguintes gêneros: Melipona, Scaptotrigona e Frieseomellita. As abelhas foram obtidas diretamente de meliponários de seis localidades distribuídas nos estados de Alagoas, Bahia e Pernambuco. As coletas foram realizadas em diferentes períodos, entre 2013 e 2014 (Tabela 1).

Tabela 1. Origem geográfica das amostras dos pólens armazenados de cinco espécies de abelhas sem ferrão.

\begin{tabular}{|c|c|c|c|}
\hline Espécie & Código & Origem geográfica & Data de coleta \\
\hline \multirow{3}{*}{$\begin{array}{c}\text { Melipona (Melipona) subnitida } \\
\text { Ducker, } 1910\end{array}$} & A1 & Mata Grande - Al & \multirow{3}{*}{$07 / 2013$} \\
\hline & A2 & \multirow{2}{*}{$\begin{array}{c}\text { Mlata Grande - AL } \\
\left(9^{\circ} 07^{\prime} 03,7^{\prime \prime} \text { S: } 37^{\circ} 43^{\prime} 57,2^{\prime \prime} \mathrm{W}\right)\end{array}$} & \\
\hline & A3 & & \\
\hline \multirow{3}{*}{$\begin{array}{c}\text { Melipona (Michmelia) scutellaris } \\
\text { Latreille, } 1811\end{array}$} & B1 & \multirow{3}{*}{$\begin{array}{c}\text { Cruz das Almas - BA } \\
\left(12^{\circ} 40^{\prime} 26,9^{\prime \prime} \text { S:3906'17,3" W) }\right.\end{array}$} & \multirow{3}{*}{$11 / 2013$} \\
\hline & B2 & & \\
\hline & B3 & & \\
\hline \multirow{10}{*}{$\begin{array}{c}\text { Melipona (Melipona) mandacaia } \\
\text { Smith, } 1863\end{array}$} & C1 & \multirow{4}{*}{$\begin{array}{c}\text { João Dourado - BA } \\
\left(11^{\circ} 12^{\prime} 14,1^{\prime \prime} \text { S: } 41^{\circ} 30^{\prime} 15,2^{\prime \prime} \mathrm{W}\right)\end{array}$} & \multirow{4}{*}{$03 / 2013$} \\
\hline & $\mathrm{C} 2$ & & \\
\hline & C3 & & \\
\hline & C4 & & \\
\hline & C5 & \multirow{4}{*}{$\begin{array}{c}\text { Uibaí - BA } \\
\left(11^{\circ} 20^{\prime} 06,7^{\prime \prime} \text { S: 4207’50,7” W) }\right.\end{array}$} & \multirow{2}{*}{ 03/2013 } \\
\hline & C6 & & \\
\hline & $\mathrm{C} 7$ & & \multirow{2}{*}{$03 / 2014$} \\
\hline & C8 & & \\
\hline & C9 & São Gabriel - BA & \multirow{2}{*}{ 03/2014 } \\
\hline & C10 & 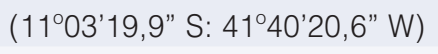 & \\
\hline \multirow{2}{*}{ Scaptotrigona sp Moure, 1942} & D1 & Canaranã - BA & \multirow{2}{*}{$03 / 2014$} \\
\hline & D2 & $\left(11^{\circ} 41^{\prime} 07,4^{\prime \prime}\right.$ S: $\left.41^{\circ} 46^{\prime} 05,3^{\prime \prime} \mathrm{W}\right)$ & \\
\hline \multirow{2}{*}{$\begin{array}{l}\text { Frieseomellita varia Lepeletier, } \\
\qquad 1836\end{array}$} & \multirow{2}{*}{ E1 } & Petrolina - PE & \multirow{2}{*}{$11 / 2013$} \\
\hline & & $\left(9^{\circ} 23^{\prime} 07,1^{\prime \prime}\right.$ S: 40³0'10,2” W) & \\
\hline
\end{tabular}


Todas as amostras foram coletadas de potes diretamente na colônia e, posteriormente, acondicionadas em vasos hermeticamente fechados e transportadas sob refrigeração para o laboratório, onde ficaram armazenadas na temperatura entre $-12{ }^{\circ} \mathrm{C} \mathrm{e}-15^{\circ} \mathrm{C}$ até a análise.

\subsection{Determinação microbiológica}

As análises microbiológicas dos pólens armazenados foram realizadas segundo a metodologia descrita em American Public Health Association (APHA) (DOWNES; ITO, 2001). Os parâmetros para qualidade comercial foram: aeróbicos mesófilos e psicotróficos, bolores e leveduras;para os indicadores de qualidade sanitária: Staphylococcus coagulase positiva, coliformes fecais e Escherichia coli; e por fim os indicadores de segurança: esporos de Clostridium sulfito redutores e Salmonella spp..

Para a realização das análises, uma alíquota de 25,0 g de cada amostra de pólen foi utilizada para a preparação da primeira diluição $\left(10^{-1}\right)$ em $225,0 \mathrm{~mL}$ de água peptonada tamponada (Himedia, Índia) a 0,1\%, e as preparações das diluições decimais subsequentes foram realizadas em tubos contendo 9,0 mL do mesmo diluente para obtenção das concentrações $10^{-2}$ e $10^{-3}$. A quantificação dos micro-organismos (aeróbios mesófilos e psicotróficos) foi realizada em placa de petri utilizando o meio de cultura Plate Count Agar- PCA (Himedia, Índia). A contagem de colônias foi efetuada após a incubação das placas a $35^{\circ} \mathrm{C}$ durante $48 \mathrm{~h}$ para mesófilo, e a $7^{\circ} \mathrm{C}$ por 10 dias para psicotróficos. Os resultados foram expressos em unidades formadoras de colônias por grama de pólen de abelha (UFC g ${ }^{-1}$ ). A quantificação de bolores e leveduras foi efetuada em Dichloran Glycerol Medium Base (Himedia, Índia), incubado a $25^{\circ} \mathrm{C}$ durante cinco dias e expressa em UFC g ${ }^{-1}$.

No estudo dos indicadores de qualidade sanitária, a determinação de Staphylococcus coagulase positiva foi realizada em Agar Baird Parker suplementado com gema de ovo e telurito de potássio 1\% (Himedia, Índia) por $24 \mathrm{~h}$ a $37{ }^{\circ} \mathrm{C}$. A quantificação de coliformes totais e Escherichia coli foi realizada com um Kit Simplate da Bio Control (FELDSINE et al., 2005). Utilizou-se a tabela de conversão SimPlate fornecida pelo fabricante, para calcular o número de coliformes e E.coli presentes nas amostras. Na avaliação de esporos de Clostridium sulfito redutor, utilizou-se $1 \mathrm{~mL}$ do caldo em suspensão nos tubos de ensaio aquecidos no banho a $80{ }^{\circ} \mathrm{C}$ durante $10 \mathrm{~min}$, que foram posteriormente plaqueados em ágar sulfito base de ferro (Himedia, Índia). Em seguida, adicionou-se outra camada de meio, e após a solidificação, foram incubados a $37^{\circ} \mathrm{C}$ durante $48 \mathrm{~h}$. A detecção de Salmonella spp. foi realizada utilizando 1-2 test (Biocontrol) como descrito no método da D'Aoust e Sewel (1988). Os resultados foram interpretados visualmente, pelo monitoramento do desenvolvimento de imunobanda, que se refere a um padrão característico de imobilização de células.

\subsection{Análises físico-química}

O teor de umidade (UMID) foi determinado em estufa

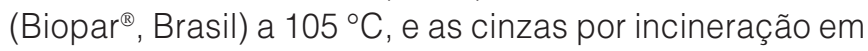
mufla (Fornitec ${ }^{\circledR}$, Brasil) a $550^{\circ} \mathrm{C}$ pelo método gravimétrico, até que atingissem o peso constante (ZENEBON et al., 2008). A atividade de água (Aw) foi realizada com medidor de atividade de água PawKit (Decagon ${ }^{\circledR}$, Washington), com temperatura de $26^{\circ} \mathrm{C}$ (ZENEBON et al., 2008). Com relação ao $\mathrm{pH}$, o mesmo foi medido na fase aquosa obtida após a mistura de $1 \mathrm{~g}$ de pólen em $75 \mathrm{~mL}$ de água destilada. A acidez total (AT) foi medida com titulação de hidróxido de sódio (Synth - Brasil) até atingir o pH 8,5 (ZENEBON et al., 2008). O teor de proteína bruta (PB) foi determinado pelo método Kjeldahl, que foi calculado utilizando o fator de conversão de 6,25 ( $N \times 6,25)$ (ZENEBON et al., 2008). $A$ análise de fibra bruta (FB) foi realizada submetendo as amostras à digestão ácida, com solução de ácido sulfúrico (Synth - Brasil) 1,25\%, seguida por digestão alcalina com hidróxido de sódio (Synth - Brasil) 1,25\% no determinador de fibra (Tecnal ${ }^{\circledR}$ TE-149, Brasil) por um período de 30 min em cada digestão (ZENEBON et al., 2008). Os lipídeos totais (LIP) foram determinados por gravimetria, com extração em hexano (Synth - Brasil) a quente no aparelho Soxhlet (Tecnal ${ }^{\circledR}$ TE-044-8, Brasil), aproximadamente por $4 \mathrm{~h}$, e utilizando dois gramas de pólens moídos (ZENEBON et al., 2008). Os açúcares redutores $(A R)$ foram determinados pelo método do ácido 3,5 dinitrosalicílico (DNS) (Synth - Brasil) (SILVA et al., 2003).

Para a determinação do teor de fenóis totais (FT) e flavonoides totais (FLT), foi obtido o extrato hidroetanólico de pólens armazenados (CARPES et al., 2009). O teor fenólico total foi estimado seguindo o método Folin-Ciocalteu (MOREIRA et al., 2008). Iniciou-se a reação de 500 uL com o extrato metanólico (MeOH) (Synth - Brasil) /pólen de abelha; $500 \mu \mathrm{L} 1: 10 \mathrm{~g} \mathrm{~mL}^{-1}$ ) e, posteriormente, se misturou com $500 \mu \mathrm{L}$ do reagente de Folin-Ciocalteu 10\% (Cromoline - Brasil) e $500 \mu \mathrm{L}$ de $\mathrm{Na}_{2} \mathrm{CO}_{3}$ (Synth - Brasil) (10\% m/v), mantendo-se no escuro à temperatura ambiente durante 1 h. Em seguida, após reação da mistura, a absorbância foi lida a 760 nm, utilizando um espectrofotômetro de UV-visível (Bel photonics ${ }^{\circledR}$-SP 2000UV) (GAEs). Para a determinação do teor de flavonoides (BARROS et al., 2010), $500 \mu \mathrm{L}$ de extratos hidroetanólicos de pólen foram misturados com $1,25 \mathrm{~mL}$ de $\mathrm{H}_{2} \mathrm{O}$ destilada e $75 \mu \mathrm{L}$ de solução de $\mathrm{NaNO}_{2}$ \% (Synth - Brasil). Depois de $5 \mathrm{~min}$, foram adicionados $150 \mu \mathrm{L}$ de uma solução $\left(\mathrm{AlCl}_{3} \cdot \mathrm{H}_{2} \mathrm{O}\right.$ Vetec - Brasil) a $10 \%$. Após 6 min, foram adicionados $500 \mu \mathrm{L}$ de $\mathrm{NaOH}(1 \mathrm{M})$ (Synth - Brasil) e $275 \mu \mathrm{L}$ de $\mathrm{H}_{2} \mathrm{O}$ destilada. A solução foi homogeneizada e a intensidade da cor foi medida a $\lambda=510 \mathrm{~nm}$. Os carboidratos (CARB) foram obtidos por diferença [100 - (cinzas + proteínas + lipídios)] (ZENEBON et al., 2008) e o valor energético ( $\mathrm{kCAL}$ ) foi estimado pela equação $=4 \times($ proteína $g+$ carboidratos g $)+9 \times($ lipídios g $)$ (ZENEBON et al., 2008). 


\subsection{Análise estatística}

Os ensaios relativos a cada parâmetro foram realizados em triplicata, conduzidos em delineamento inteiramente casualizado. As correlações lineares entre os parâmetros foram avaliadas pela correlação de Pearson $(p<0,05)$. A análise discriminante foi aplicada com o objetivo de determinar funções discriminantes usando os parâmetros que separassem os grupos. A análise discriminante (DA) é um procedimento de classificação que maximiza a variância entre as categorias e minimiza a variância dentro das categorias. A análise de componentes principais (ACP) foi realizada para determinar a relação entre o samburá das espécies e os parâmetros físico-químicos analisados. A análise hierárquica de clusters (AC) foi realizada para determinar o número de grupos formados de acordo com as similaridades entre as amostras. Todas as análises foram realizadas utilizando-se o programa $R$ versão 3.3.0 $R$ (R DEVELOPMENT CORE TEAM, 2016).

\section{Resultados e discussão}

\subsection{Contaminantes microbiológicos}

As bactérias mesófilas estavam presentes em todas as amostras analisadas, com médias entre 1,8 a 8,5 × 102 UFC. $g^{-1}$. Entretanto, apenas para os pólens armazenados da espécie Frieseomellita varia $\left(4,2 \times 10^{1} \mathrm{UFC} \mathrm{g}^{-1}\right)$ foi possível enumerar bolores e leveduras. Também não foram registradas a presença de Salmonella spp., esporos de Clostridium sulfito redutores, coliformes totais, Escherichia coli, Staphylococcus coagulase positiva em todas as amostras, conforme indicado na Tabela 2.

Verificou-se que os níveis de quantificação para os parâmetros de qualidade comercial (aeróbios mesófilos, bolores e leveduras) estão abaixo do código alimentar argentino (ARGENTINA, 1990) que determina valores máximos de $150 \times 10^{3} \mathrm{UFC} . g^{-1}$ e $10^{2} \mathrm{UFC} . g^{-1}$ para pólens apícolas, respectivamente. A contagem para esse grupo de micro-organismo foi relatada em diversos estudos para pólen apícola desidratado (DE-MELO et al., 2015; ESTEVINHO et al., 2012; FEÁS et al., 2012; NOGUEIRA et al., 2012). Embora a presença de micro-organismos mesófilos não esteja associada ao risco à saúde dos consumidores, sugere-se que tais alimentos não suportem um longo tempo de armazenamento in natura, podendo assim acarretar prejuízo econômico. Os micro-organismos indicadores de segurança (Salmonella spp. e esporos de Clostridium sulfito redutores), bem como os indicadores de qualidade sanitária (Escherichia coli e Staphylococcus coagulase positiva) estavam ausentes em todas as amostras, e esses dados corroboram com os estudos realizados (BÁRBARA et al., 2015) para pólens armazenados frescos por Melipona mandacaia. A Resolução da Diretoria Colegiada, RDC 12/2001 (BRASIL, 2001a) descreve sobre padrões microbiológicos para alimentos e estabelece a ausência de Salmonella spp. em $25 \mathrm{~g}$ dos alimentos; deste modo, todas as amostras encontraram-se dentro dos padrões estabelecidos por esta resolução.

Embora não existam critérios microbiológicos no regulamento para a produção de pólen no Brasil, são necessários estudos sobre as condições e seguranças microbiológicas, bem como o estabelecimento de padrões internacionais de qualidade. Estas medidas são necessárias, tendo em vista que o pólen é utilizado em países em desenvolvimento como suplemento alimentar (PETROVIĆ et al., 2014).

\subsection{Composição físico-química}

Estão expostos na Tabela 3 os valores médios para parâmetros físico-químicos analisados: teor de umidade, cinzas, atividade de água (Aw), valor de pH, acidez total, proteínas, lipídios, fibras, teor de fenóis totais, teor de flavonoides e açúcares redutores.

Com relação à análise de correlação (Tabela 4), a mesma permitiu conhecer a relação entre todos os parâmetros. Observou-se uma correlação altamente significativa entre a umidade e a maioria dos parâmetros avaliados, assim como uma correlação positiva entre fenóis e flavonoides. No entanto, o par ( $\mathrm{pH}$ com AT) foi o que apresentou menor correlação, sendo considerado como negativo e não significativo.

Tabela 2. Resultados das análises microbiológicas para amostras de pólen armazenado de cinco espécies de abelhas sem ferrão do Nordeste do Brasil.

\begin{tabular}{cccccc} 
Espécie & $(\mathbf{n})$ & $\begin{array}{c}\text { Coliformes Totais } \\
\left(\mathbf{N M P . g ^ { - 1 } )}\right.\end{array}$ & $\begin{array}{c}\text { Bolores e } \\
\text { Leveduras } \\
\left(\mathbf{U F C . g ^ { - 1 } )}\right.\end{array}$ & $\begin{array}{c}\text { Psicotróficos } \\
\left.\text { (UFC. } \mathbf{g}^{-1}\right)\end{array}$ & $\begin{array}{c}\text { Mesófilos } \\
\left(\mathbf{U F C} . \mathbf{g}^{-1}\right)\end{array}$ \\
Melipona subnitida & 3 & $<3$ & $<1,0 \times 10$ & $<1 \times 10$ & $2,5 \times 10^{2}$ \\
M. sclutellaris & 3 & $<3$ & $<1,0 \times 10$ & $<1 \times 10$ & $1,8 \times 10^{2}$ \\
M. mandacaia & 10 & $<3$ & $<1,0 \times 10$ & $<1 \times 10$ & $5,4 \times 10^{2}$ \\
Scaptotrigona sp. & 2 & $<3$ & $<1,0 \times 10$ & $<1 \times 10$ & $8,5 \times 10^{2}$ \\
Frieseomellita varia & 1 & $<3$ & $4,2 \times 10^{1}$ & $<1 \times 10$ & $4,0 \times 10^{2}$ \\
\hline
\end{tabular}

$(n)=$ Número de amostra por espécie, UFC=Unidade formadora de colônia e NMP=Número mais provável. 
Tabela 3. Médias e desvio padrão das variáveis físico-químicas de pólen armazenado de abelha sem ferrão do Nordeste do Brasil.

\begin{tabular}{crrrrrrr} 
Variáveis & M. subnitida & \multicolumn{1}{c}{ M.sclutellaris } & M. mandacaia & Scaptotrigona sp. & \multicolumn{1}{c}{ F. varia } & Mínimo & Máximo \\
UMIDADE & $30,44 \pm 2,72$ & $50,05 \pm 1,12$ & $34,52 \pm 1,00$ & $28 \pm 0,38$ & $23,17 \pm 0,01$ & 23,17 & 50,05 \\
CINZA & $5,54 \pm 0,51$ & $4,21 \pm 0,26$ & $4,94 \pm 0,35$ & $5,23 \pm 1,05$ & $4,4 \pm 0,33$ & 4,21 & 5,54 \\
Aw & $0,86 \pm 0,02$ & $0,94 \pm 0,01$ & $0,86 \pm 0,01$ & $0,82 \pm 0,01$ & $0,76 \pm 0,01$ & 0,76 & 0,94 \\
pH & $3,51 \pm 0,04$ & $3,88 \pm 0,08$ & $3,50 \pm 0,05$ & $3,71 \pm 0,05$ & $3,9 \pm 0,02$ & 3,50 & 3,90 \\
AT & $158,28 \pm 4,09$ & $182,89 \pm 9,32$ & $141,57 \pm 4,88$ & $83,25 \pm 19,65$ & $84,5 \pm 2,29$ & 83,25 & 182,89 \\
PB & $23,19 \pm 2,64$ & $30,37 \pm 1,52$ & $21,68 \pm 1,62$ & $15,98 \pm 2,82$ & $16,28 \pm 0,5$ & 15,98 & 30,37 \\
FB & $3,56 \pm 0,49$ & $2,13 \pm 0,19$ & $2,66 \pm 1,18$ & $9,9 \pm 2,46$ & $3,07 \pm 0,21$ & 2,13 & 9,90 \\
LIP & $4,21 \pm 0,8$ & $5,99 \pm 0,28$ & $4,29 \pm 1,46$ & $2,29 \pm 0,21$ & $1,45 \pm 0,35$ & 1,45 & 5,99 \\
AR & $13,64 \pm 2,74$ & $16,87 \pm 1,46$ & $13,01 \pm 2,94$ & $22,92 \pm 1,9$ & $31,21 \pm 0,10$ & 13,01 & 31,21 \\
FT & $80,43 \pm 3,93$ & $381,17 \pm 24,53$ & $78,85 \pm 8,75$ & $80,23 \pm 8,86$ & $71,77 \pm 1,18$ & 71,77 & 381,17 \\
FLT & $9,22 \pm 3,67$ & $55,4 \pm 7,53$ & $7,40 \pm 1,65$ & $9,27 \pm 0,4$ & $6,90 \pm 0,04$ & 6,90 & 55,40 \\
CARB & $63,5 \pm 3,35$ & $57,3 \pm 1,49$ & $66,44 \pm 2,51$ & $66,6 \pm 1,9$ & $74,8 \pm 1,18$ & 57,30 & 74,80 \\
kCAL & $384,65 \pm 4,13$ & $404,59 \pm 2,7$ & $391,03 \pm 9,03$ & $350,94 \pm 13,25$ & $377,37 \pm 2,46$ & 350,94 & 404,59 \\
\hline
\end{tabular}

Aw=Atividade de água, AT=Acidez livre, PB=Proteína bruta, FB=Fibra bruta, LIP=Lipídios, AR=Açúcar redutor, FT=Fenóis totais, FLT =Flavonoide totais, $\mathrm{CARB}=$ Carboidrato, $\mathrm{kCAL}=$ Valor energético.

Tabela 4. Coeficientes de correlação simples (com p valores) entre as variáveis físico-químicas de pólens armazenados de abelhas sem ferrão do Nordeste do Brasil.

\begin{tabular}{|c|c|c|c|c|c|c|c|c|c|c|c|c|c|}
\hline & UMIDADE & CINZA & Aw & pH & AT & PB & FB & LIP & AR & FT & FLT & CARB & kCAL \\
\hline UMIDADE & 1,00 & & & & & & & & & & & & \\
\hline CINZA & $-0,52^{\star *}$ & 1,00 & & & & & & & & & & & \\
\hline Aw & $0,91^{* *}$ & $-0,36^{*}$ & 1,00 & & & & & & & & & & \\
\hline $\mathrm{pH}$ & $0,38^{* *}$ & $-0,34^{*}$ & $0,15^{\text {ns }}$ & 1,00 & & & & & & & & & \\
\hline AT & $0,75^{\star \star}$ & $-0,26^{n s}$ & $0,82^{\star *}$ & $-0,04^{\mathrm{ns}}$ & 1,00 & & & & & & & & \\
\hline PB & $0,81^{* \star}$ & $-0,38^{\star \star}$ & $0,79^{\star \star}$ & $0,25^{\mathrm{ns}}$ & $0,86^{\star \star}$ & 1,00 & & & & & & & \\
\hline FB & $-0,40^{* \star}$ & $0,41^{\star *}$ & $-0,37^{\star *}$ & $0,19^{\text {ns }}$ & $-0,67^{\star *}$ & $-0,57^{\star *}$ & 1,00 & & & & & & \\
\hline LIP & $0,63^{*}$ & $-0,16^{n s}$ & $0,58^{\star *}$ & $0,05^{\text {ns }}$ & $0,66^{\star *}$ & $0,68^{\star *}$ & $-0,42^{\star *}$ & 1,00 & & & & & \\
\hline AR & $-0,29^{\star *}$ & $-0,16^{n s}$ & $-0,47^{\star \star}$ & $0,59^{\star * *}$ & $-0,53^{\star \star}$ & $-0,23^{\text {ns }}$ & $0,31^{*}$ & $-0,34^{*}$ & 1,00 & & & & \\
\hline FT & $0,89^{* *}$ & $-0,51^{\star *}$ & $0,75^{\star *}$ & $0,69^{\star *}$ & $0,61^{\star *}$ & $0,76^{\star *}$ & $-0,24^{\mathrm{ns}}$ & $0,50^{\star *}$ & $0,08^{\text {ns }}$ & 1,00 & & & \\
\hline FLT & $0,88^{* *}$ & $-0,52^{\star *}$ & $0,74^{\star *}$ & $0,68^{\star *}$ & $0,59^{\star *}$ & $0,73^{\star *}$ & $-0,20^{n s}$ & $0,47^{\star *}$ & $0,07^{\text {ns }}$ & $0,99^{\star *}$ & 1,00 & & \\
\hline CARB & $-0,71^{\star *}$ & $0,07^{n s}$ & $-0,71^{\star *}$ & $-0,32^{*}$ & $-0,66^{\star *}$ & $-0,84^{\star *}$ & $0,10^{\text {ns }}$ & $-0,75^{\star *}$ & $0,19^{\text {ns }}$ & $-0,71^{\star *}$ & $-0,68^{\star *}$ & 1,00 & \\
\hline $\mathrm{KCAL}$ & $0,63^{* *}$ & $-0,48^{\star *}$ & $0,57^{\star *}$ & $-0,04^{\text {ns }}$ & $0,78^{\star *}$ & $0,74^{\star *}$ & $-0,89^{\star *}$ & $0,77^{\star *}$ & $-0,33^{*}$ & $0,47^{\star \star}$ & $0,43^{* *}$ & $-0,44^{\star *}$ & 1,00 \\
\hline
\end{tabular}

ns não significativo; * significativo a $5 \%(p<0,05) ;{ }^{* *}$ significativo a $1 \%(p<0,01)$. Aw=Atividade de água, AT= Acidez livre, PB=Proteína bruta, $\mathrm{FB}=$ Fibra bruta, $\mathrm{LIP}=\mathrm{Lipídios,} \mathrm{AR}=\mathrm{Açúcar}$ redutor, $\mathrm{FT}=$ Fenóis totais, $\mathrm{FLT}=\mathrm{Flavonoide}$ totais, $\mathrm{CARB}=\mathrm{Carboidrato}$, $\mathrm{kCAL}=\mathrm{Valor}$ energético.

Para completar os estudos, a análise discriminante foi realizada para determinar previamente os parâmetros físico-químicos mais significativos na caracterização dos pólens armazenados das espécies de abelhas sem ferrão. Optou-se pelo método de análise discriminante passo a passo (stepwise discriminant analysis), o qual adiciona ou remove variáveis do modelo. Desta forma, obtiveram-se 10 parâmetros mais significativos entre os 13 investigados, tais como: UMID, cinza, Aw, pH, AT, FB, AR, FT, FLT, CARB. Vale ainda ressaltar que esses parâmetros são capazes de distinguir os três grupos de pólens.

Também se utilizou a análise de clusters (AC), que foi aplicada para os dados autoescalonados, empregando-se a distância euclidiana para calcular as similaridades das amostras e um procedimento de agrupamento hierárquico para estabelecer os clusters. Os resultados obtidos podem ser observados no dendograma da Figura 1, em que se verifica três grupos distintos: um primeiro grupo formado

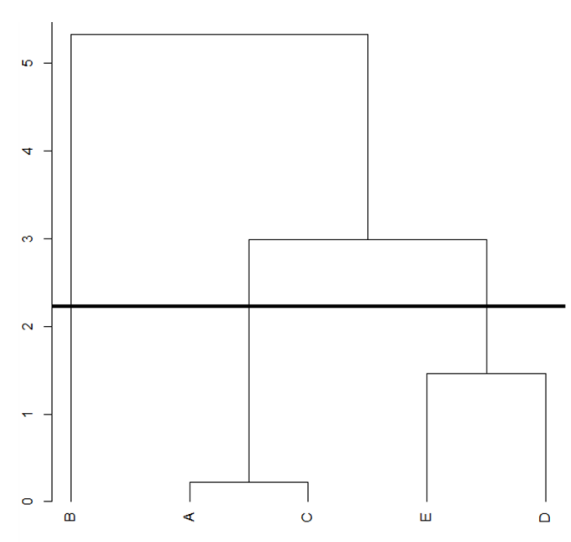

Figura 1. Dendograma com as médias dos parâmetros físico-químicos de pólen armazenado de abelha sem ferrão, Nordeste, Brasil. $A=M$. subnitida, $B=M$. scutellaris, $C=M$. mandacaia, $\mathrm{D}=$ Scaptotrigona $\mathrm{sp}, \mathrm{E}=F$. varia. 

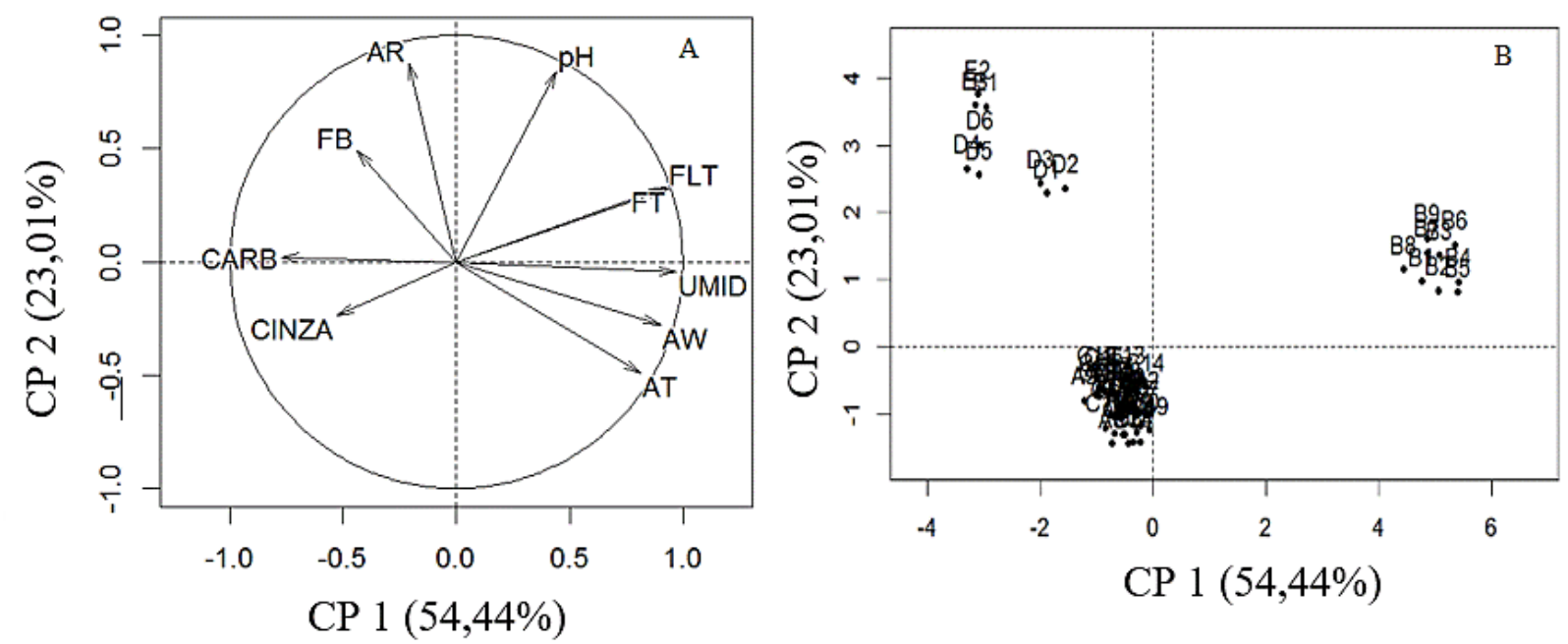

Figura 2. Análise de Componentes Principais. A - Considerando os parâmetros após analise discriminante. B - Formação dos grupos com base nas espécies de abelhas sem ferrão. Legenda: $A=M$. subnitida, $B=M$. scutellaris, $C=M$. mandacaia, $D=$

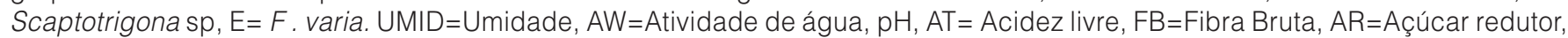
$\mathrm{FT}=$ Fenóis totais, $\mathrm{FLT}=$ Flavonoide totais, $\mathrm{CARB}=$ Carboidrato.

apenas pela espécie B; um segundo grupo pelas espécies A e $\mathrm{C}$ e um terceiro grupo formado pelas espécies $\mathrm{D}$ e $\mathrm{E}$.

A componente principal foi utilizada para estudar as relações dos parâmetros físico-químicos dos pólens armazenados por diferentes espécies de abelhas sem ferrão. De fato, os resultados obtidos a partir das análises discriminantes mostraram que dois componentes principais foram responsáveis por $77,45 \%$ da variância total das características. Portanto, o primeiro componente principal explicou $54,44 \%$ da variância total, ao passo que o segundo componente principal foi responsável por $23,01 \%$ da variação total das amostras (Figura 2), enquanto os demais apresentaram pouca importância para obter $100 \%$ da variância total dos dados. As amostras de pólens armazenados da espécie B foram mais ricas em fenóis, flavonoides e umidade; mas, por outro lado apresentaram menor teor de carboidratos e cinzas. As amostras das espécies $D$ e $E$ foram agrupadas com base nos teores de açúcares redutores e fibra bruta, que indicaram valores mais elevados, porém com baixa atividade de água. Por fim, as amostras das espécies A e C apresentaram mais variáveis em comum, sendo determinante para formação do grupo as variáveis de acidez total, cinzas e carboidratos.

De acordo com a análise de componentes principais, foram obtidos resultados semelhantes aos encontrados na análise de cluster. Empregando-se dados autoescalonados nesta análise, observou-se a formação de três grupos bem definidos, caracterizando uma diferença entre os parâmetros físico-químicos dos pólens armazenados por abelhas sem ferrão e suas respectivas espécies.
Com relação ao teor de umidade, as espécies $\mathrm{A}$, B e C apresentaram valores superiores a $30 \%$, sendo maiores do que o limite estabelecido pelo Regulamento Técnico Brasileiro (RTB) para pólen apícola no Brasil (BRASIL, 2001b). No entanto, esses valores corroboram com os valores encontrados por Bárbara et al. (2015).

O valor médio das cinzas para todas as espécies foi superior ao estabelecido no Código de Alimentos da Argentina (ARGENTINA, 1990) e também no RTB para pólen apícola. No entanto, Campos et al. (2008) estabeleceram alguns padrões para pólen apícola, com parâmetros superiores aos estabelecidos pela legislação, considerando valores ideais para cinzas entre $2 \%$ a $5 \%$.

Com relação aos valores de acidez $(83,25$ a 182,28 mol. $\left.\mathrm{kg}^{-1}\right)$, fibra bruta $(2,13 \%$ a $9.9 \%)$ e proteína bruta ou total $(15,98 \%$ a $30,27 \%)$, todos estiveram de acordo com o RT para pólen apícola no Brasil (BRASIL, 2001b). Em estudos realizados com pólens armazenados por abelhas sem ferrão no Amazonas e na Venezuela, Vit et al. (2016) relataram valores de proteínas bruta ou total inferiores aos encontrados neste estudo.

Os resultados mostraram que entre os pólens das cinco espécies de abelhas sem ferrão estudadas, apenas o pólen da espécie $F$. varia apresentou teor de lipídios abaixo de $2 \%$. Os valores de $\mathrm{pH}$ estavam entre 3,5 a 3,9 para todas as amostras de pólens das diferentes espécies, o que está em desacordo com o estabelecido pelo RTB para pólen apícola no Brasil. De acordo com Vásquez e Olofsson, (2009), as reduções no pH durante a conversão dos pólens coletados pelas abelhas em pólen apícola ou pólen armazenado foram atribuídas às atividades das 
bactérias produtoras de ácido lático. Essas bactérias são normalmente adicionadas ao pólen a partir do estômago e do mel das abelhas. Para o conteúdo de carboidratos (57,30\% a 74,8\%) e valor energético (350,94 a 404,59 kcal), ambos corroboraram com os resultados apresentados para os pólens armazenados por abelhas sem ferrão do gênero Melipona no estado do Amazonas (REBELO et al., 2016). Quanto aos valores de compostos fenólicos totais e flavonoides, foi observada grande variação entre as amostras de $M$. scutellaris e demais espécies. Como exemplo, o pólen da espécie $M$. scutellaris apresentou valores cinco vezes mais elevados quando comparados ao de outras espécies. Valores inferiores aos encontrados neste estudo foram relados por Vit et al. (2016) e Imarkiewicz-Żukowska et al. (2013).

O pólen da espécie M. scutellaris formou um grupo isolado com outras espécies do gênero Melipona, porque apresentou características físico-químicas peculiares e sugere-se que a origem geográfica possa ter contribuído para a formação deste grupo. Porém, os pólens das espécies $M$. subnitida e M. mandacaia formaram um único grupo, pois apresentaram características semelhantes e ambas as espécies estão inseridas na região semiárida. Por fim, o terceiro grupo foi formado pelas amostras de pólens armazenados de Scaptotrigona sp e F. varia.

Tendo em vista que a composição do pólen não possui um padrão rigoroso, a causa desta variabilidade deve-se à origem floral, áreas geográficas, condições ambientais e climáticas, idade e estado nutricional das plantas e espécies de abelhas. De fato, o pólen, dentre outros produtos da colmeia, normalmente apresenta maior variação (NOGUEIRA et al., 2012; ESWARAN; BHARGAVA, 2014).

\section{Conclusão}

As amostras dos pólens armazenados pelas cinco espécies de abelhas sem ferrão estudadas apresentam boas condições microbiológicas, sem contaminação por micro-organismos patogênicos. Verificou-se a possibilidade de separação dos pólens das cinco espécies de abelhas sem ferrão em três grupos, com base nos parâmetros físico-químico avaliados. O resultado desse estudo reforça a necessidade de adequação do Regulamento Técnico existente para PA, para que venha abranger os pólens armazenados das espécies de abelhas sem ferrão. Dessa forma, haverá maior facilidade para a comercialização do samburá, agregando valor e gerando renda aos meliponicultores.

\section{Agradecimentos}

Os autores agradecem o Programa Nacional de Pós-doutorado (PNPD), da Coordenação de Aperfeiçoamento de Pessoal de Nível Superior (CAPES) pelo auxílio financeiro (28022017), bolsa de pós-doutorado (C. S. Machado), e pelo apoio da bolsa de estudo
(M. F. Santa Barbara). Os autores também agradecem a Universidade Federal do Recôncavo da Bahia (UFRB) pela estrutura física e contribuição nas análises e o Conselho Nacional de Desenvolvimento Científico e Tecnológico (CNPq) pela bolsa de pesquisador (305228/2013-7) de C. A. L. de Carvalho.

\section{Referências}

ARGENTINA. Ministerio da la Salud. Administración Nacional de Medicamentos, Alimentos y Tecnología Médica. Decreto 2126, 30 de Junio de 1971. Reglamentario de la Ley 18.284, que establece la vigencia de las normas higiénico-sanitarias, bromatológicas y de identificación comercial contenidas en el Código Alimentario Argentino. Capitulo 10: Alimentos azucarados. Artículo 785, Boletin Oficial Republica Argentina, Buenos Aires, 12 dez. 1990.

BÁRBARA, M. S.; MACHADO, C. S.; SODRÉ, G. D. S.; DIAS, L. G.; ESTEVINHO, L. M.; CARVALHO, C. A. L. Microbiological Assessment, Nutritional Characterization and Phenolic Compounds of Bee Pollen from Mellipona mandacaia Smith, 1983. Molecules, Switzerland, v. 20, n. 7, p. 12525-12544, 2015. http://dx.doi. org/10.3390/molecules200712525.

BARROS, L.; HELENO, S. A.; CARVALHO, A. M.; FERREIRA, I. C. F. R. Lamiaceae often used in Portuguese folk medicine as a source of powerful antioxidants: Vitamins and phenolics. Lebensmittel-Wissenschaft + Technologie, v. 43, n. 3, p. 544-550, 2010. http://dx.doi.org/10.1016/j.Iwt.2009.09.024.

BRASIL. AGÊNCIA NACIONAL DE VIGILÂNCIA SANITÁRIA - ANVISA. Resolucao RDC n 12, de 02 de janeiro de 2001. Aprova regulamento técnico sobre os padrões microbiológicos para alimentos. Diário Oficial [da] República Federativa do Brasil, Brasilia, DF, 10 jan. 2001a.

BRASIL. Ministério da Agricultura, Pecuária e Abastecimento. Secretaria de Defesa e Agropecuária. Instrução Normativa ${ }^{\circ}$ 03, de 19 de janeiro de 2001. Aprova os regulamentos técnicos de identidade e qualidade de apitoxina, cera de abelha, geleia real, geleia real liofilizada, pólen apícola, própolis e extrato de própolis. Diário Oficial [da] República Federativa do Brasil, Brasília, DF, 23 jan. 2001 b.

CAMARGO, J. M. F.; PEDRO, S. R. M. 2013. Meliponini Lepeletier, 1836. In: MOURE, J. S., URBAN, D. \& MELO, G. A. R. (Orgs). Catalogue of bees (Hymenoptera, Apoidea) in the neotropical region. Disponível em: http://www.moure.cria.org.br/catalogue. Acesso em: 29 ago. 2017

CAMPOS, M. G.; BOGDANOV, S.; DE ALMEIDA-MURADIAN, L. B.; SZCZESNA, T.; MANCEBO, Y.; FRIGERIO, C.; FERREIRA, F. Pollen composition and standardisation of analytical methods. Journal of Apicultural Research, v. 47, n. 2, p. 154-161, 2008. http://dx.doi.org/10.1080/00218839.2008.11101443. 
Caracterizações microbiológica e físico-química de pólens armazenados por abelhas sem ferrão

Bárbara, M. F. S. et al.

CARPES, S. T.; CABRAL, I. S.; LUZ, C. F. P.; CAPELETTI, J. P.; ALENCAR, S. M.; MASSON, M. L. Palynological and physicochemical characterization of Apis mellifera $\mathrm{L}$. bee pollen in the Southern region of Brazil. Journal of Food Agriculture and Environment, v. 7, p. 667-673, 2009.

CHANTARUDEE, A.; PHUWAPRAISIRISAN, P.; KIMURA, K.; OKUYAMA, M.; MORI, H.; KIMURA, A.; CHANCHAO, C. Chemical constituents and free radical scavenging activity of corn pollen collected from Apis mellifera hives compared to floral corn pollen at Nan, Thailand. BMC Complementary and Alternative Medicine, v. 12, n. 45, p. 1-12, 2012. Disponível em: <https://bmccomplementalternmed.biomedcentral.com/ track/pdf/10.1186/1472-6882-12-45>. PMid:22513008.

D'AOUST, J. Y.; SEWELL, A. M. Reliability of the immunodiffusion 1-2 Test ${ }^{\mathrm{TM}}$ system for detection of Salmonella in foods. Journal of Food Protection, v. 51, n. 11, p. 853-856, 1988. http://dx. doi. org/10.4315/0362-028X-51.11.853.

DE FLORIO ALMEIDA, J.; REIS, A. S.; HELDT, L. F. S.; PEREIRA, D.; BIANCHIN, M.; MOURA, C.; CARPES, S. T. Lyophilized bee pollen extract: a natural antioxidant source to prevent lipid oxidation in refrigerated sausages. Lebensmittel-Wissenschaft + Technologie, v. 76, p. 299-305, 2017. http://dx.doi.org/10.1016/j. Iwt.2016.06.017.

DE-MELO, A. A. M.; ESTEVINHO, M. L. M. F.; ALMEIDAMURADIAN, L. B. A diagnosis of the microbiological quality of dehydrated bee-pollen produced in Brazil. Letters in Applied Microbiology, v. 61, n. 5, p. 477-483, 2015. http://dx.doi. org/10.1111/lam.12480. PMid:26280091.

DENISOW, B.; DENISOW-PIETRZYK, M. Biological and therapeutic properties of bee pollen: a review. Journal of the Science of Food and Agriculture, v. 96, n. 13, p. 4303-4309, 2016. http:// dx.doi.org/10.1002/jsfa.7729. PMid:27013064.

DOWNES, F. P.; ITO, K. Compendium of methods for the microbiological examination of foods. Washington: APHA, 2001. http://dx.doi.org/10.2105/9780875531755.

ESTEVINHO, L. M.; RODRIGUES, S.; PEREIRA, A. P.; FEÁS, $X$. Portuguese bee pollen: palynological study, nutritional and microbiological evaluation. International Journal of Food Science \& Technology, v. 47, n. 2, p. 429-435, 2012. http:// dx.doi.org/10.1111/j.1365-2621.2011.02859.x.

ESWARAN, V. U.; BHARGAVA, H. R. Chemical analysis and anti-microbial activity of Karnataka bee bread of apis species. World Applied Sciences Journal, v. 32, n. 3, p. 379-385, 2014.

FEÁS, X.; VÁZQUEZ-TATO, M. P.; ESTEVINHO, L.; SEIJAS, J. A.; IGLESIAS, A. Organic bee pollen: botanical origin, nutritional value, bioactive compounds, antioxidant activity and microbiological quality. Molecules, Switzerland, v. 17, n. 7, p. 8359-8377, 2012. http://dx.doi.org/10.3390/molecules17078359. PMid:22785265.
FELDSINE, P. T.; LIENAU, A. H.; ROA, N. H.; GREEN, S. T. Enumeration of total coliforms and $E$. coli in foods by the SimPlate ${ }^{\circledR}$ coliform and E. coli color indicator method and conventional culture methods: collaborative study. Journal of AOAC International, v. 88, n. 5, p. 1318-1333, 2005. PMid:16385981.

IMARKIEWICZ-ŻUKOWSKA, R.; NALIWAJKO, S. K.; BARTOSIUK, E.; MOSKWA, J.; ISIDOROV, V. Chemical composition and antioxidant activity of beebread, and its influence on the glioblastoma cell line (U87MG). Journal of Apicultural Science, v. 57, n. 2, p. 147-157, 2013. http://dx.doi.org/10.2478/jas-2013-0025.

ISIDOROV, V. A.; BAGAN, R.; BAKIER, S.; SWIECICKA, I. Chemical composition and antimicrobial activity of Polish herbhoneys. Food Chemistry, v. 171, p. 84-88, 2015. http:// dx.doi.org/10.1016/j.foodchem.2014.08.112. PMid:25308646.

MOREIRA, L.; DIAS, L. G.; PEREIRA, J. A.; ESTEVINHO, L. Antioxidant properties, total phenols and pollen analysis of propolis samples from Portugal. Food and Chemical Toxicology, v. 46, n. 11, p. 3482-3485, 2008. http://dx.doi.org/10.1016/j. fct.2008.08.025. PMid:18804144.

NOGUEIRA, C.; IGLESIAS, A.; FEÁS, X.; ESTEVINHO, L. M. Commercial bee pollen with different geographical origins: a comprehensive approach. International Journal of Molecular Sciences, v. 13, n. 9, p. 11173-11187, 2012. http://dx.doi. org/10.3390/ijms130911173. PMid:23109845.

NOGUEIRA-NETO, P. Vida e criação de abelhas indígenas sem ferrão. São Paulo: Nogueirapis, 1997.

OMAR, W. A. W.; AZHAR, N. A.; FADZILAH, N. H.; KAMAL, N. N. S. N. M. Bee pollen extract of Malaysian stingless bee enhances the effect of cisplatin on breast cancer cell lines. Asian Pacific Journal of Tropical Biomedicine, v. 6, n. 3, p. 265-269, 2016. http://dx.doi.org/10.1016/j.apjtb.2015.12.011.

PETROVIĆ, T.; NEDIĆ, N.; PAUNOVIĆ, D.; RAJIĆ, J.; MATOVIĆ, K.; RADULOVIĆ, Z.; KRNJAJA, V. Natural mycobiota and aflatoxin B1 presence in bee pollen collected in Serbia. Biotechnology in Animal Husbandry, v. 30, n. 4, p. 731-741, 2014. http:// dx.doi.org/10.2298/BAH1404731P.

R DEVELOPMENT CORE TEAM. A language and environment for statistical computing. Vienna: R Foundation for Statistical Computing, 2013. Disponível em: http://www.r-project.org. Acesso em: 22 jun. 2016.

REBElO, K. S.; FERREIRA, A. G.; AL CARVALHO-ZILSE, G. Physicochemical characteristics of pollen collected by Amazonian stingless bees. Ciência Rural, v. 46, n. 5, p. 927-932, 2016. http://dx.doi.org/10.1590/0103-8478cr20150999.

SILVA, R. N., MONTEIRO, V. N., ALCANFOR, J. D. X., ASSIS, E. M., ASQUIERI, E. R. Comparision methods for the determination 
Caracterizações microbiológica e físico-química de pólens armazenados por abelhas sem ferrão Bárbara, M. F. S. et al.

of reducer's sugars and total in honey. Food Science and Technology, Campinas, v. 23, p. 337-341, 2003.

VÁSQUEZ, A.; OLOFSSON, T. C. The lactic acid bacteria involved in the production of bee pollen and bee bread. Journal of Apicultural Research, v. 48, n. 3, p. 189-195, 2009. http:// dx.doi.org/10.3896/IBRA.1.48.3.07.

VIT, P. Melipona favosa pot-honey from Venezuela. In: VIT, P.; PEDRO, S. R. M.; ROUBIK, D. Pot-honey. New York: Springer, 2013. p. 363-373.
VIT, P.; SANTIAGO, B.; PEDRO, S. R.; PEREZ-PEREZ, E.; PENAVERA, M. Chemical and bioactive characterization of pot-pollen produced by Melipona and Scaptotrigona stingless bees from Paria Grande, Amazonas State, Venezuela. Emirates Journal of Food and Agriculture, v. 28, n. 2, p. 78-84, 2016. http:// dx.doi.org/10.9755/ejfa.2015-05-245.

ZENEBON, O.; PASCUET, N. S.; TIGLEA, P. (Ed.) Métodos físico-químicos para análise de alimentos. São Paulo: Instituto Adolfo Lutz, 2008. 1020 p. 